VOL. 60 (1999) [245-251]

\title{
RECALCITRANCE IN GROUPS
}

\author{
R.G. Burns, W.N. Herfort, S.-M. Kam, O. Macedońska and P.A. Zalesskil
}

\section{To B.H. Neumann on his 90th birthday}

Motivated by a well-known conjecture of Andrews and Curtis, we consider the question as to how in a given $n$-generator group $G$, a given set of $n$ "annihilators" of $G$, that is, with normal closure all of $G$, can be transformed by standard moves into a generating $n$-tuple. The recalcitrance of $G$ is defined to be the least number of elementary standard moves ("elementary M-transformations") by means of which every annihilating $n$-tuple of $G$ can be transformed into a generating $n$-tuple. We show that in the classes of finite and soluble groups, having zero recalcitrance is equivalent to nilpotence, and that a large class of 2-generator soluble groups has recalcitrance at most 3 . Some examples and remarks are included.

\section{INTRODUCTION}

We shall understand an $n$-generator group to be a group that can be generated by $n$ elements, but not fewer, and an $n$-annihilator group to be one that is the normal closure of $n$ elements, but not fewer. We shall call a set $\left\{r_{1}, \ldots, r_{n}\right\}$ of elements of a group $G$ whose normal closure is the whole of $G$ an annihilating $n$-tuple for $G$, and write $\left\langle r_{1}, \ldots, r_{n}\right\rangle^{G}=G$. These include, of course, generating $n$-tuples $\left\{g_{1}, \ldots, g_{n}\right\}$, for which we write $\left\langle g_{1}, \ldots, g_{n}\right\rangle=G$.

We are interested here in the question as to whether, and if so then with what degree of difficulty, an annihilating $n$-tuple $\left\{r_{1}, \ldots, r_{n}\right\}$ of a given group $G$ can be transformed via natural elementary moves into a generating $n$-tuple. The "elementary moves" as defined by Andrews and Curtis in [1] are Nielsen transformations supplemented by the transformations replacing an $r_{i}$ by a conjugate $r_{i}{ }^{g}$. In [2, Proposition 1], it was shown essentially that the result of any finite sequence of such transformations can be achieved by means of a sequence of transformations of the form

$$
\left\{r_{1}, \ldots, r_{n}\right\} \longrightarrow\left\{r_{1}, \ldots, r_{i} w, \ldots, r_{n}\right\}, w \in\left\langle r_{1}, \ldots, r_{i-1}, r_{i+1}, \ldots, r_{n}\right\rangle^{G}
$$

called elementary M-transformations ( $\mathrm{M}$ for "modulo" since $r_{i}$ is replaced by any element congruent to it modulo the other $r_{j} s$ ), and conversely. For some purposes - see

Received 12th May, 1999

Copyright Clearance Centre, Inc. Serial-fee code: 0004-9727/99 \$A2.00+0.00. 
[2] and the remarks below - the latter transformations are more convenient than those of Andrews and Curtis, and it is these that we shall take as our "elementary moves" here.

Let $G$ be an $n$-generator, $n$-annihilator group. We define the recalcitrance of an annihilating $n$-tuple for $G$ to be the least number of elementary M-transformations required to transform that $n$-tuple into a generating $n$-tuple. (If it is not possible to so transform the given annihilating $n$-tuple, we say that its recalcitrance is infinite.) We then define the recalcitrance of the group $G$ to be the largest of the recalcitrances of all of its annihilating $n$-tuples.

Our main results are as follows. In Section 2 we consider groups of recalcitrance zero, showing that at least in the classes of finite and soluble groups having zero recalcitrance is equivalent to nilpotence. In Section 2, by way of giving examples we examine the recalcitrance of two particular groups, namely the infinite dihedral group $\mathbb{Z}_{2} * \mathbb{Z}_{2}$ and the wreath product $\mathbb{Z} \backslash \mathbb{Z}$. Finally, in Section 3 , we prove that a great many 2 -generator, 2 -annihilator soluble groups, including all free soluble groups of rank 2 , have recalcitrance at most 3 .

REMARKS. 1. The concept of recalcitrance has its origin in the paper [1] of Andrews and Curtis, which contains the by now well-known conjecture that in the free group $F_{n}$ of rank $\mathrm{n}$ every annihilating $n$-tuple has finite recalcitrance. Some candidates for counterexamples, that is, for having infinite recalcitrance, are given in [2]. One formerly hopeful such candidate (not in [2]), namely the annihilating pair $\left\{y x y x^{-1} y^{-1} x^{-1}, x^{2} y^{-3}\right\}$ for $F_{2}$, the free group on $x, y$, has recently been shown by computer to have finite recalcitrance after all [3]. That in fact this pair has recalcitrance 2 can be shown as follows. Write briefly

$$
r:=y x y x^{-1} y^{-1} x^{-1}, s:=x^{2} y^{-3} \text {. }
$$

Then $r=y x^{-1} x^{2} y x^{-1} y^{-3} y^{2} x^{-1}$, whence

$$
r \equiv y x^{-1} y x^{-1} y^{2} x^{-1} \bmod \langle s\rangle^{F_{2}} .
$$

Thus as our first elementary M-transformation we may replace $\{r, s\}$ by $\left\{r_{1}, s\right\}$, where $r_{1}:=y x^{-1} y x^{-1} y^{2} x^{-1}$. Now $r_{1}$ is primitive (that is, a member of a generating pair for $F_{2}$ ) since it is conjugate to $\left(y x^{-1}\right)^{3} y$, which is clearly primitive. Hence one further elementary M-transformation will yield a pair $\left\{r_{1}, s_{1}\right\}$ of generators of $F_{2}$.

2. In [2] the term "complexity" was used to mean something very close to recalcitrance. The present version of the concept seems preferable, in particular since it avoids negative numbers. Thus in terms of recalcitrance, Theorem 1 of [2] asserts that the annihilating pair $\left\{x^{3} y^{-1} x^{-2} y, y^{3} x^{-1} y^{-2} x\right\}$ for $F_{2}$ has recalcitrance $\geqslant 3$. 
3. The concept of an elementary M-transformation lends itself to the formulation of an analogue of the concept of recalcitrance - and hence also of the Andrews-Curtis conjecture - for Lie rings. Thus if $L$ denotes the free Lie ring on $x_{1}, \ldots, x_{n}$, and the ideal generated by $r_{1}, \ldots, r_{n}$ is all of $L$, an elementary $M$-transformation of $\left\{r_{1}, \ldots, r_{n}\right\}$ changes this to an $n$-tuple of the form $\left\{r_{1}, \ldots, r_{i}+w, \ldots, r_{n}\right\}$ where $w$ belongs to the ideal generated by $\left\{r_{1}, \ldots, r_{i-1}, r_{i+1}, \ldots, r_{n}\right\}$. The recalcitrance of such an $n$-tuple $\left\{r_{1}, \ldots, r_{n}\right\} \subset L$ is now defined as before. Question: Is there a connection between the original Andrews-Curtis conjecture for $F_{n}$ and its analogue for the Lie ring constructed from the lower central series of $F_{n}$ in the usual way?

4. The concept of recalcitrance might be extended to arbitrary $n$-generator groups (that is, not necessarily $n$-annihilator) by considering ordered rather than unordered annihilating $n$-tuples. We have assumed our groups are $n$-annihilator as well as $n$ generator chiefly for the sake of simplicity.

\section{ZERO RECALCITRANCE}

Most of the material of this section amounts to a paraphrase of known facts.

For an $n$-generator, $n$-annihilator group to have zero recalcitrance simply means that every set of $n$ elements whose normal closure is $G$, actually generates $G$. It is well known - and not too difficult to prove by induction on the nilpotency class - that nilpotent groups have this property:

PROPOS ITION. Every finitely generated nilpotent group has zero recalcitrance.

We shall now show that, at least among finite and soluble groups, zero recalcitrance in fact characterises nilpotence. The following result is also probably known in one form or another.

TheOREM 1. Let $G$ be an $n$-generator, $n$-annihilator group. Then $G$ has zero recalcitrance if and only if the Frattini subgroup $\Phi(G)$ contains the commutator subgroup $[G, G]$ of $G$.

COROLLARY. An n-generator, $n$-annihilator finite or soluble group has recalcitrance zero if and only if it is nilpotent.

In the finite case this follows via Wielandt's result that a finite group $G$ is nilpotent if and only if $[G, G] \leqslant \Phi(G)$ (see for example, [4, p.132]). For the soluble case, see [4, p.460, Exercise 3].

Proof of Theorem 1: Assume first that $\Phi(G) \geqslant[G, G]$, and let $\left\{a_{1}, \ldots, a_{n}\right\}$ be any annihilating $n$-tuple for $G$. We shall show that such an $n$-tuple actually generates $G$.

Since the cosets $a_{1}[G, G], a_{2}[G, G], \ldots, a_{n}[G, G]$ generate $G /[G, G]$ and $G$ is finitely generated, there is a generating set for $G$ of the form $\left\{a_{1} c_{1}, \ldots, a_{n} c_{n}, c_{n+1}, \ldots, c_{n+k}\right\}$, 
where $c_{1}, \ldots, c_{n+k} \in[G, G]$. Hence certainly

$$
\left\langle a_{1}, a_{2}, \ldots, a_{n}, c_{1}, c_{2}, \ldots, c_{n+k}\right\rangle=G .
$$

However since we are assuming that $\Phi(G) \geqslant[G, G]$, and since, as is well known, $\Phi(G)$ consists of the elements of $G$ omissible from every generating set for $G$, it follows that $\left\langle a_{1}, \ldots, a_{n}\right\rangle=G$.

For the converse suppose that in $G$ we always have

$$
\left\langle a_{1}, \ldots, a_{n}\right\rangle^{G}=G \Longrightarrow\left\langle a_{1}, \ldots, a_{n}\right\rangle=G .
$$

Let $\left\{g_{1}, \ldots, g_{n}\right\}$ be any generating $n$-tuple for $G$. We first show that for any elements $c_{1}, \ldots, c_{n} \in[G, G]$ the set $\left\{g_{1} c_{1}, \ldots, g_{n} c_{n}\right\}$ also generates $G$. For this it suffices to show that for an arbitrary element $c_{1}$ of $[G, G]$ we have $\left\langle g_{1} c_{1}, \ldots, g_{n}\right\rangle=G$; the desired conclusion then follows via $n$ iterations. Now since $G /\left(g_{2}, \ldots, g_{n}\right)^{G}$ is cyclic, and therefore certainly Abelian, we must have $c_{1} \in\left(g_{2}, \ldots, g_{n}\right)^{G}$. Hence

$$
\left\langle g_{1} c_{1}, g_{2}, \ldots, g_{n}\right\rangle^{G} \geqslant\left\langle g_{1}, g_{2}, \ldots, g_{n}\right\rangle=G
$$

whence by our assumption $\left\langle g_{1} c_{1}, g_{2}, \ldots, g_{n}\right\rangle=G$.

Now let $c$ be any element of $[G, G]$, and let $X$ be any subset of $G$ such that $\langle X, c\rangle=G$. Since $X$ generates $G$ modulo $[G, G]$, there exist elements $x_{1}, \ldots, x_{n} \in$ $\langle X\rangle$ and $d_{1}, \ldots, d_{n} \in[G, G]$ such that $g_{1}=x_{1} d_{1}, \ldots, g_{n}=x_{n} d_{n}$, that is, $x_{1}=$ $g_{1} d_{1}^{-1}, \ldots, x_{n}=g_{n} d_{n}^{-1}$. Hence by our earlier argument $\left\langle x_{1}, \ldots, x_{n}\right\rangle=G$, so that certainly $\langle X\rangle=G$. Thus every element $c \in[G, G]$ is omissible, whence $[G, G] \leqslant$ $\Phi(G)$.

\section{Two EXAmples}

3.1. The infinite dihedral group $\mathbb{Z}_{2} * \mathbb{Z}_{2}$ has recalcitrance 1 . Presented by $D:=$ $\left\langle a, b \mid a^{2}=b^{2}=1\right\rangle$, the infinite dihedral group may be considered as consisting of all finite strings of alternating $a$ 's and $b$ 's. Since $D /[D, D] \cong \mathbb{Z}_{2} \times \mathbb{Z}_{2}$, generated by $a[D, D]$ and $b[D, D]$, any annihilating pair must have one of the forms

$$
\{a c, b d\},\{a c, a b d\}, \text { or }\{b c, a b d\},
$$

for some $c, d \in[D, D]=\left\langle(a b)^{2}\right\rangle$. Since each element of the form $a c$ or $b c$ is a member of a generating pair, namely $\{a c, a b\}$ or $\{b c, a b\}$, it follows that one elementary Mtransformation suffices to transform each of the three types of annihilating pairs into a generating pair. 
3.2. The restricted wreath product $\mathbb{Z} \imath \mathbb{Z}$. It is not difficult to verify that this group has recalcitrance $\geqslant 2$, for example, by observing that if $x$ generates the top group and $y$ a coordinate subgroup, then the pair $\left\{x^{2} y^{3}, x^{3} y^{4}\right\}$ is annihilating, but neither of its elements is a member of any generating pair (since setting either of them equal to 1 results in a non-cyclic quotient of the wreath product). On the other hand by our main theorem below, $\mathbb{Z} \imath \mathbb{Z}$ has recalcitrance $\leqslant 3$. Thus its recalcitrance is either 2 or 3 . We leave it to the reader to decide which.

\section{Soluble groups}

We show here that a great many 2 -generator soluble groups, including all rank-2 free soluble groups, have recalcitrance $\leqslant 3$. (This puts paid to the suggestion made in [2, Remark 6] that as a possible approach to disproving the Andrews-Curtis conjecture one might try to show that the recalcitrance of some annihilating pair of the rank- 2 free soluble group goes to $\infty$ with the solubility length.)

THEOREM 2. Let $G$ be a 2 -generator soluble group with commutator quotient $G /[G, G]$ free Abelian of rank 2 (that is, $\cong \mathbb{Z} \times \mathbb{Z}$ ). Then $G$ has recalcitrance $\leqslant 3$.

REMARK. At the expense of some combinatorial complexity, our method of proof can certainly be extended to yield a bound for the recalcitrance of an $\boldsymbol{n}$-generator soluble group (with commutator quotient free Abelian of rank $n$ ). On the other hand, we have not been able to dispense with the condition on the commutator quotient, although it seems unlikely that this is at all germane.

Corollary. The free soluble groups of rank 2 (that is, $F_{2} / F_{2}^{(k)}$, where $F_{2}^{(k)}$ is the $k$ th commutator subgroup of $F_{2}$ ) have recalcitrance $\leqslant 3$ (that is, independently of $k)$.

PROOF OF THE THEOREM: Let $\{r, s\}$ be any annihilating pair for $G$. The assumption that $G /[G, G]$ be free Abelian of rank 2 ensures that there exists a generating pair $\{a, b\}$ for $G$ and elements $u, v \in[G, G]$ such that

$$
r=a u, \quad s=b v .
$$

This can be established by means of the following well-known argument. Let $f: F_{2} \rightarrow G$ be any epimorphism, and let $\rho, \sigma$ be preimages under $f$ of $r, s$ respectively. Let $\{x, y\}$ be any free basis for $F_{2}$, and write $\bar{x}:=x\left[F_{2}, F_{2}\right], \bar{y}:=y\left[F_{2}, F_{2}\right]$. For some integers $k, l, m, n$ we have

$$
\rho \equiv x^{k} y^{l} \bmod \left[F_{2}, F_{2}\right], \quad \sigma \equiv x^{m} y^{n} \bmod \left[F_{2}, F_{2}\right]
$$

Since the cosets $\rho\left[F_{2}, F_{2}\right], \sigma\left[F_{2}, F_{2}\right]$ generate $F_{2} /\left[F_{2}, F_{2}\right]$, there is a Nielsen transformation taking $(\bar{x}, \bar{y})$ to $\left(\bar{x}^{k} \bar{y}^{l}, \bar{x}^{m} \bar{y}^{n}\right)$. Lifting this Nielsen transformation to $F_{2}$ yields an 
automorphism of $F_{2}$ taking $(x, y)$ to a pair $(\alpha, \beta)$ of free generators of $F_{2}$, satisfying $\alpha \equiv \rho \bmod \left[F_{2}, F_{2}\right], \beta \equiv \sigma \bmod \left[F_{2}, F_{2}\right]$. Applying $f$ to these congruences then yields the desired result (1) with $a:=f(\alpha), b:=f(\beta)$.

We define in the usual way "commutators of weight $w$ in $a$ and $b$ " by induction on $w$. For $w=1$ these are taken to be just $a^{ \pm 1}, b^{ \pm 1}$. A commutator of weight $w>1$ in $a$ and $b$ is then defined to be an element of $G$ expressible as $\left[c_{1}, c_{2}\right]\left(:=c_{1}^{-1} c_{2}^{-1} c_{1} c_{2}\right)$ where $c_{1}, c_{2}$ are respectively commutators of weights $w_{1}, w_{2}<w$ in $a$ and $b$, such that $w_{1}+w_{2}=w$. It is well-known, and not difficult to prove using the standard group identities

$$
\begin{gathered}
x y=y x[x, y], \quad[x, y]^{-1}=[y, x], \\
{[x y, z]=[x, z][[x, z], y][y, z],} \\
{[x, y z]=[x, z][x, y][[x, y], z],}
\end{gathered}
$$

that every element of $[G, G]$ can be expressed as a product of finitely many commutators of weights $\geqslant 2$ in $a$ and $b$. Thus in particular in (1) $u$ and $v$ can be so expressed, say

$$
u=c_{1} c_{2} \ldots c_{m}, \quad v=d_{1} d_{2} \ldots d_{n} .
$$

Now suppose that $G$ has solubility length $k$; this means simply that commutators of weight $k$ commute with each other. If $c(a, b)$ is any commutator of weight $w \geqslant 2$ in $a$ and $b$, then $c(a, b) \equiv c\left(u^{-1}, b\right) \bmod \langle a u\rangle^{G}$, that is, the relation $c(a, b)=c\left(u^{-1}, b\right)$ is a consequence of the relation $a u=1$. (Here $c\left(u^{-1}, b\right)$ is intended to denote the result of replacing each entry $a^{ \pm 1}$ in the commutator $c(a, b)$ by $u^{\mp 1}$.) Using the identities (2) we can now expand $c\left(u^{-1}, b\right)$ as a product of commutators of weights strictly greater than the weight of the original commutator $c(a, b)$. Carrying out this substitution for each of $c_{1}, \ldots, c_{m}$ in (3), we obtain an element $a c_{1}^{\prime} c_{2}^{\prime} \ldots c_{l}^{\prime} \equiv 1 \bmod \langle r\rangle^{G}$, where

$$
\min _{1 \leqslant i \leqslant m}\left\{\text { weight } c_{i}\right\}<\min _{i \leqslant j \leqslant l}\left\{\text { weight } c_{j}^{\prime}\right\} .
$$

Iteration of this procedure will eventually yield an element

$$
a \widehat{u}=a \widehat{c}_{1} \ldots \widehat{c}_{p} \equiv 1 \bmod \langle r\rangle^{G},
$$

where $\widehat{c}_{1}, \ldots \widehat{c}_{p}$ are commutators in $a$ and $b$, all of weight $\geqslant k+2$.

Suppose that some $\widehat{c}_{i}=\widehat{c}_{i}(a, b)$ is such that as a commutator in $a$ and $b$ at least two of its entries are $a^{ \pm 1}$. Then on replacing all such entries by $\widehat{u}^{\mp 1}$, we shall obtain the identity element: $\widehat{c}\left(\widehat{u}^{-1}, b\right)=1$ since commutators of weights $\geqslant k$ commute in $G$. Hence $\widehat{c}_{i}(a, b) \equiv 1 \bmod \langle r\rangle^{G}$. Performing this substititution for all $\widehat{c}_{i}$ with at least two entries $a^{ \pm 1}$, and leaving the others as they are, we obtain

$$
a \tilde{u}=a \tilde{c}_{1} \ldots \tilde{c}_{q} \equiv 1 \bmod \langle r\rangle^{G},
$$


where $\tilde{c}_{1}, \ldots, \tilde{c}_{q}$ are expressible as commutators of weights $\geqslant k+2$ in $a$ and $b$, all of which have just one entry of the form $a^{ \pm 1}$. Since their weights are $\geqslant k+2$, they must then each have at least two entries of the form $b^{ \pm 1}$.

We are now ready to apply our first elementary M-transformation to the given annihilating pair $\{r, s\}=\{a u, b v\}$. In $b v=b d_{1} \ldots d_{n}$, we replace each commutator $d_{i}(a, b)$ by $d_{i}\left(\widetilde{u}^{-1}, b\right)$; in view of (4) these are congruent to each other modulo $\langle r\rangle^{G}$. As before, any $d_{i}(a, b)$ with two or more entries equal to $a^{ \pm 1}$ will under this substitution yield the identity element. It follows that this elementary M-transformation transforms $\{r, s\}$ into $\{r, b \tilde{v}\}$ where $\tilde{v}$ has the form $\tilde{v}=\tilde{d}_{1} \ldots \tilde{d}_{t}$ with each $\widetilde{d}_{j}$ expressible as a commutator in $a$ and $b$ of weight $\geqslant k+2$ with at least two entries of the form $b^{ \pm 1}$. Replacement of such entries by $\widetilde{v}^{\mp 1}$ will then result in

$$
b \tilde{d}_{1}\left(a, \widetilde{v}^{-1}\right) \tilde{d}_{2}\left(a, \widetilde{v}^{-1}\right) \ldots \tilde{d}_{t}\left(a, \widetilde{v}^{-1}\right) \equiv 1 \bmod \langle b \widetilde{v}\rangle^{G} .
$$

However since each $\tilde{d}_{j}\left(a, \widetilde{v}^{-1}\right)=1$, we infer that in fact. $b \in\langle b \widetilde{v}\rangle^{G}$. For the second elementary M-transformation, applied now to $\{r, b \widetilde{v}\}$, we replace $b$ by 1 everywhere in $r=a u(a, b)$ (as we may since $b \in\langle b \tilde{v}\rangle^{G}$ ), thereby obtaining the pair $\{a, b \tilde{v}\}$. As the third and final elementary M-transformation we replace $a$ by $l$ in $b \tilde{v}(a, b)$, obtaining $\{a, b\}$.

\section{REFERENCES}

[1] J.J. Andrews and M.L. Curtis, 'Extended Nielsen operations in free groups', Amer. Math. Monthly 73 (1966), 21-28.

[2] R.G. Burns and O. Macedonska, 'Balanced presentations of the trivial group', Bull. London Math. Soc. 25 (1993), 513-526.

[3] A.D. Miasnikov, 'Genetic algorithms and the Andrews-Curtis conjecture', (preprint).

[4] D.J.S. Robinson, $A$ course in the theory of groups (Springer-Verlag, Berlin, Heidelberg, New York, 1982).

Department of Mathematics and Statistics

York University

Toronto

Canada M3J 1P3

Kwi Shing Estate

New Territories

Hong Kong

China

Department of Mathematics

University of Brasilia

70910-900 Brasilia-DF

Brazil
Institut für Angew. Mathematik

Technische Universität

A-1040 Vienna

Austria

Institute of Mathematics

Silesian Technical University

44-100 Gliwice

Poland 